\title{
Is There a Role for Multiple Lines of Anti-HER2 Therapies Administered Beyond Progression in HER2- Mutated Non-Small Cell Lung Cancer? A Case Report and Literature Review
}

\author{
Giulio Metro (D) - Sara Baglivo · Riccardo Moretti - Guido Bellezza • \\ Angelo Sidoni · Fausto Roila
}

Received: May 18, 2020 / Published online: July 7, 2020

(C) The Author(s) 2020

\begin{abstract}
Oncogene-addicted non-small cell lung cancer (NSCLC) comprises a number of distinct disease subtypes, each of which is characterised by druggable genetic alterations. Among them, the receptor tyrosine kinase protein human epidermal receptor 2 (HER2) is occasionally found deregulated via gene mutation and/or amplification and/or protein overexpression. HER2 mutation, in particular, is a relatively rare condition which occurs in $1-4 \%$ of NSCLC patients,
\end{abstract}

Digital Features To view digital features for this article, go to https://doi.org/10.6084/m9.figshare. 12514373.

G. Metro $(\bowtie) \cdot$ F. Roila

Medical Oncology, Santa Maria Della Misericordia Hospital, Azienda Ospedaliera Di Perugia, Perugia, Italy

e-mail: giulio.metro@yahoo.com

S. Baglivo

Laboratory of Oncology, Medical Oncology, Santa

Maria Della Misericordia Hospital, Azienda

Ospedaliera Di Perugia, Perugia, Italy

R. Moretti

Department of Radiology, Santa Maria Della

Misericordia Hospital, Azienda Ospedaliera Di

Perugia, Perugia, Italy

\section{G. Bellezza · A. Sidoni}

Division of Pathology and Histology, Department of Experimental Medicine, University of Perugia

Medical School, Perugia, Italy especially in those with adenocarcinoma histology and a never/light smoking history. However, the clinical relevance of a HER2 mutation in NSCLC relies on the fact that this genetic alteration has been associated with sensitivity to anti-HER2 therapies such as the monoclonal antibody trastuzumab or the panHER-tyrosine kinase inhibitor poziotinib. Here we describe the case of a NSCLC patient with an activating exon 20 G776VinsC mutation in the HER2 gene who responded well to multiple lines of trastuzumab-based therapies administered beyond progression and poziotinib given sequentially. In this specific case, the discovery of a druggable genetic alteration such as a mutation in the HER2 gene allowed for longterm control of the disease through the use of highly effective anti-HER2 therapies.

Keywords: Beyond progression; HER2 mutation; Non-small cell lung cancer; Poziotinib; Trastuzumab 


\section{Key Summary Points}

Human epidermal receptor 2 (HER2) can be aberrantly expressed in non-small cell lung cancer (NSCLC) through a variety of mechanisms, including HER2 mutation and/or amplification and/or protein overexpression.

HER2 mutations are found in $1-4 \%$ of NSCLC patients, and they may have sensitivity to anti-HER2 therapies such as monoclonal antibodies, antibody-drug conjugates and small-molecule tyrosine kinase inhibitors (TKIs).

We describe the case of a NSCLC patient with an activating exon 20 G776VinsC HER2 mutation who responded well to multiple lines of combination treatment with the anti-HER2 antibody trastuzumab administered beyond progression as well as to the pan-HER-TKI poziotinib given sequentially.

This case highlights the importance of detecting a HER2 mutation in patients with advanced NSCLC as this might positively affect the treatment scenario through the use of effective anti-HER2 therapies.

\section{INTRODUCTION}

The human epidermal growth factor receptor (HER2) is a member of the epidermal growth factor receptor (EGFR) family, which also includes EGFR, HER3 and HER4 [1]. Each receptor comprises an extracellular ligandbinding domain, a transmembrane segment and an intracellular portion with tyrosine kinase activity. In cancer cells, HER2 can promote tumour growth and survival through homo- and heterodimerization with other receptors of the EGFR family, which in turn activates intracellular signalling pathways such as the raf/mitogen-activated protein kinase (MAPK) and phosphatidylinositol-4,5-bisphosphate 3-kinase (PI3K)/AKT cascades [1]. No natural ligand has been identified for HER2, which is consistent with the fact that this receptor acts as the preferred partner of dimerization of other receptors of the EGFR family. Importantly, HER2 can be found deregulated via gene mutation and/or amplification and/or protein overexpression in multiple malignancies, including breast cancer, gastric cancer and non-small cell lung cancer (NSCLC) [1]. In NSCLC in particular, HER2 mutations are present in $1-4 \%$ of patients with lung adenocarcinoma, especially never smokers. Of note, HER2 mutation is mutually exclusive with other driver mutations such as EGFR, anaplastic lymphoma kinase $(A L K)$, ROS proto-oncogene 1 receptor tyrosine kinase (ROS1) and v-raf murine sarcoma viral oncogene homolog $\mathrm{B} 1$ (BRAF) $[1,2]$. Even more importantly, HER2-mutated NSCLC patients appear to derive benefit from anti-HER2 therapies such as monoclonal antibodies, antibody-drug conjugates and smallmolecule tyrosine kinase inhibitors (TKIs). However, in some cases, conflicting results have been reported in clinical trials of HER2-mutated NSCLCs treated with anti-HER2 therapies, and the role of anti-HER2 therapies in this context is not fully understood [1].

In the present case report, we tackle the concept of continuous HER2 blockade in NSCLC by describing a HER2-mutated NSCLC patient who benefited from multiple lines of anti-HER2 therapies administered beyond progression.

\section{CASE}

In January 2014, a 42-year-old never-smoker woman who had been complaining of a dry cough for 2 months was diagnosed with a $2-\mathrm{cm}$ large lesion of the medium lobe of the lung. Biopsy of this lesion obtained during bronchoscopy was compatible with TTF1-positive adenocarcinoma. A PET/CT scan was positive for the presence of multiple metastatic ipsilateral mediastinal lymph nodes and a single metastasis in the contralateral lung, which 
configured T2N2M1-stage IV disease. As molecular biology was negative for EGFR mutation and $A L K$ gene rearrangement, the patient started first-line chemotherapy with cisplatin/pemetrexed, of which she received four cycles with partial response followed by pemetrexed maintenance. In August 2014, disease progression in the right lung was observed at a PET/CT scan, and the patient received stereotactic radiotherapy on the primary lung lesion (62 Gy, 25 fractions). After radiotherapy to the lung, systemic therapy was halted and the patient was followed up with serial PET/CT scans every 3 months until May 2015, when a new PET/CT scan showed progressive disease in the left lung. A new 1-cm-large lesion in the left occipital region of the brain with no neurological symptoms was also detected at a brain MRI. At that time, since the patient was a young never-smoker woman who was negative for both EGFR and $A L K$, we carried out sequential molecular testing for select genetic drivers including HER2. Direct sequencing of the HER2 gene revealed the presence of a G776VinsC mutation (Fig. 1). This mutation configured a disease potentially responding to anti-HER2 therapies, so the patient was managed thereafter with multiple lines of anti-HER2 therapies (see Fig. 2 for a timeline of significant events).
After receiving stereotactic radiotherapy (20 Gy, single fraction) to the new brain lesion, the patient resumed pemetrexed in combination with the anti-HER2 monoclonal antibody trastuzumab given at $8 \mathrm{mg} / \mathrm{kg}$ loading dose followed by $6 \mathrm{mg} / \mathrm{kg}$, both drugs being administered every 3 weeks. The choice of resuming pemetrexed was based on the fact that we aimed to further exploit a histologic-specific cytotoxic agent active in lung adenocarcinoma. In October 2015, a PET/CT scan was compatible with complete extracranial response, while an MRI showed disease stability of the brain lesion. The patient continued pemetrexed plus trastuzumab until February 2016 when a new PET/CT scan showed extracranial disease progression in both lungs. As a result, it was decided to continue tri-weekly trastuzumab and change the companion cytotoxic drug by switching to weekly paclitaxel. Importantly, a new PET/CT scan performed on June 2016 showed partial response at extracranial sites of disease that lasted for approximately 1 year until May 2017, when a PET/TC scan showed disease progression in both lungs. At that time, the patient had to withdraw from anti-tumour treatment because of the development of severe mitral insufficiency secondary to bacterial endocarditis, which was managed surgically with mitral valve

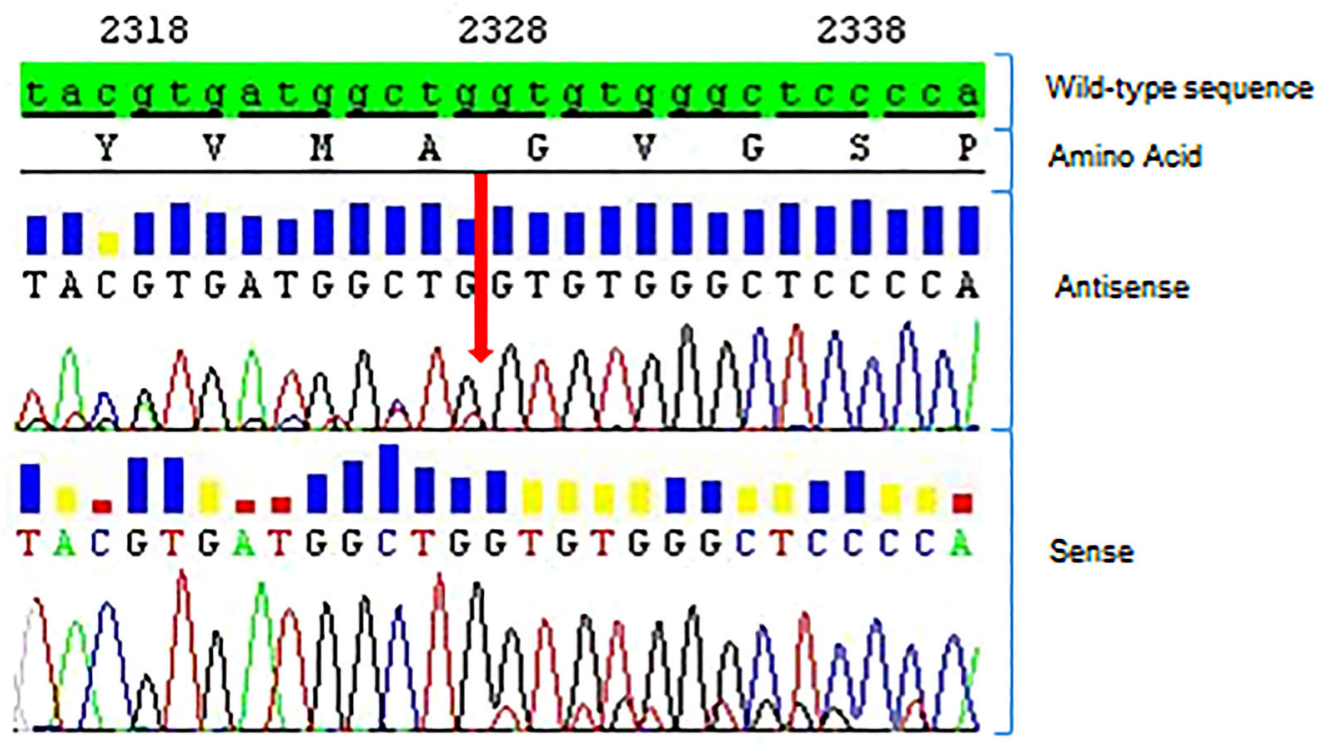

Fig. 1 Diagnostic of HER2 mutation (patient no. TP35). Sanger sequencing read with heterozygous HER2 exon 20 insertion: c.2326_2327insTTT, p.(G776VinsC) (red arrow) 


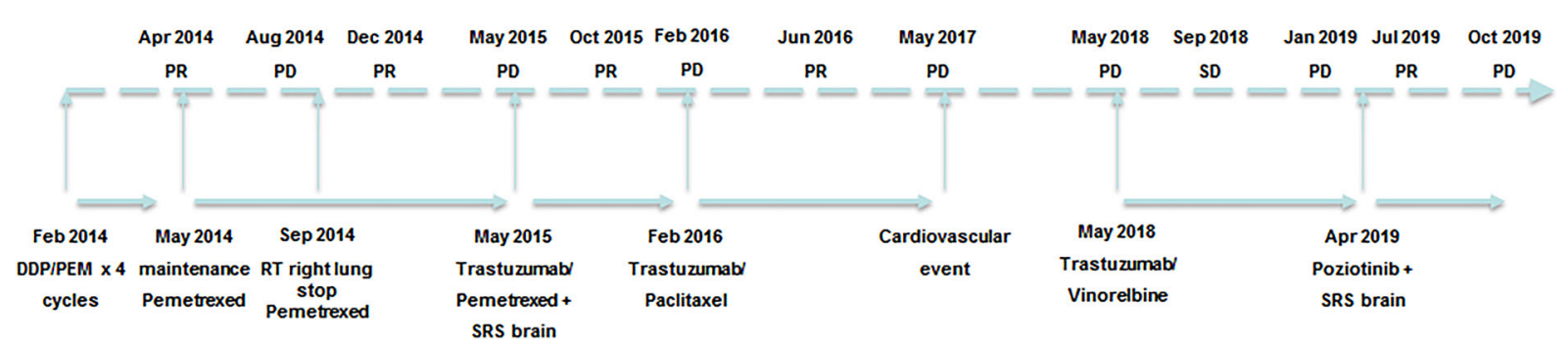

Fig. 2 Patient timeline. $D D P / P E M$ cisplatin/pemetrexed, $P D$ progressive disease, $P R$ partial response, $S R S$ stereotactic radiosurgery

repair. At recovery, a new restaging through a PET/TC scan on May 2018 showed further disease progression in the lung, with a new MRI showing stable disease in the brain. As the mitral insufficiency was not deemed to be trastuzumab-related, after careful discussion with the patient, a decision was made to resume triweekly trastuzumab with a change in the companion cytotoxic with the introduction of oral vinorelbine on days 1 and 8 every 3 weeks. A new PET/CT scan performed after 4 months showed stable disease at extracranial sites. In January 2019, a PET/CT scan revealed disease progression in the lung, while an MRI documented disease progression in the brain with the onset of a new centimetric lesion in the right cerebellum. In April 2019, following radiosurgery to the new brain lesion (20 Gy, single fraction), the patient started treatment with the pan-HER-TKI poziotinib at the dose of $16 \mathrm{mg}$ daily p.o. within an expanded access program, from which she obtained a partial response in the lung. In October 2019, the disease progressed again in the lung and brain with the onset of multiple brain lesions. The patient then received whole brain radiotherapy (30 Gy, 10 fractions) and started chemotherapy with carboplatin/gemcitabine, which is still ongoing as of January 2020. Written informed consent to publish the patient's case was provided by the patient in an anonymous form.

\section{DISCUSSION}

Our case shows that HER2 mutation in lung cancer is a druggable target as the patient experienced a significant clinical benefit from multiple anti-HER2 treatments, namely trastuzumab-based therapies beyond progression and the pan-HER-TKI poziotinib (Fig. 2), with a prolonged survival greater than 6 years. In HER2-positive breast cancer defined at immunohistochemistry (IHC) by a score of $3+$ or $2+$ and FISH positivity, the administration of multiple lines of trastuzumab-based therapies beyond progression has shown effectiveness with a potential improvement in survival $[3,4]$. However, the administration of multiple antiHER2 therapies in HER2-mutated NSCLC is largely unexplored, which renders the present case very interesting. Importantly, pemetrexed rechallenge with the addition of trastuzumab was associated with clinical benefit in our patient despite the fact that the patient experienced prior disease progression while on pemetrexed maintenance. Although data coming from other malignancies such as HER2positive breast and gastric cancers suggest that trastuzumab exerts its maximal efficacy when administered in combination with chemotherapy, it is likely that in this specific case response to treatment could be largely due to trastuzumab treatment $[5,6]$. Importantly, in studies of HER2-mutated NSCLC, trastuzumab has mostly been used in combination with chemotherapy. Table 1 lists selected studies evaluating antiHER2 monoclonal antibodies and antibody-drug conjugates for the treatment of HER2-mutated NSCLC.

The concept of continuing target inhibition at the time of disease progression, with or without the addition of chemotherapy, is not 


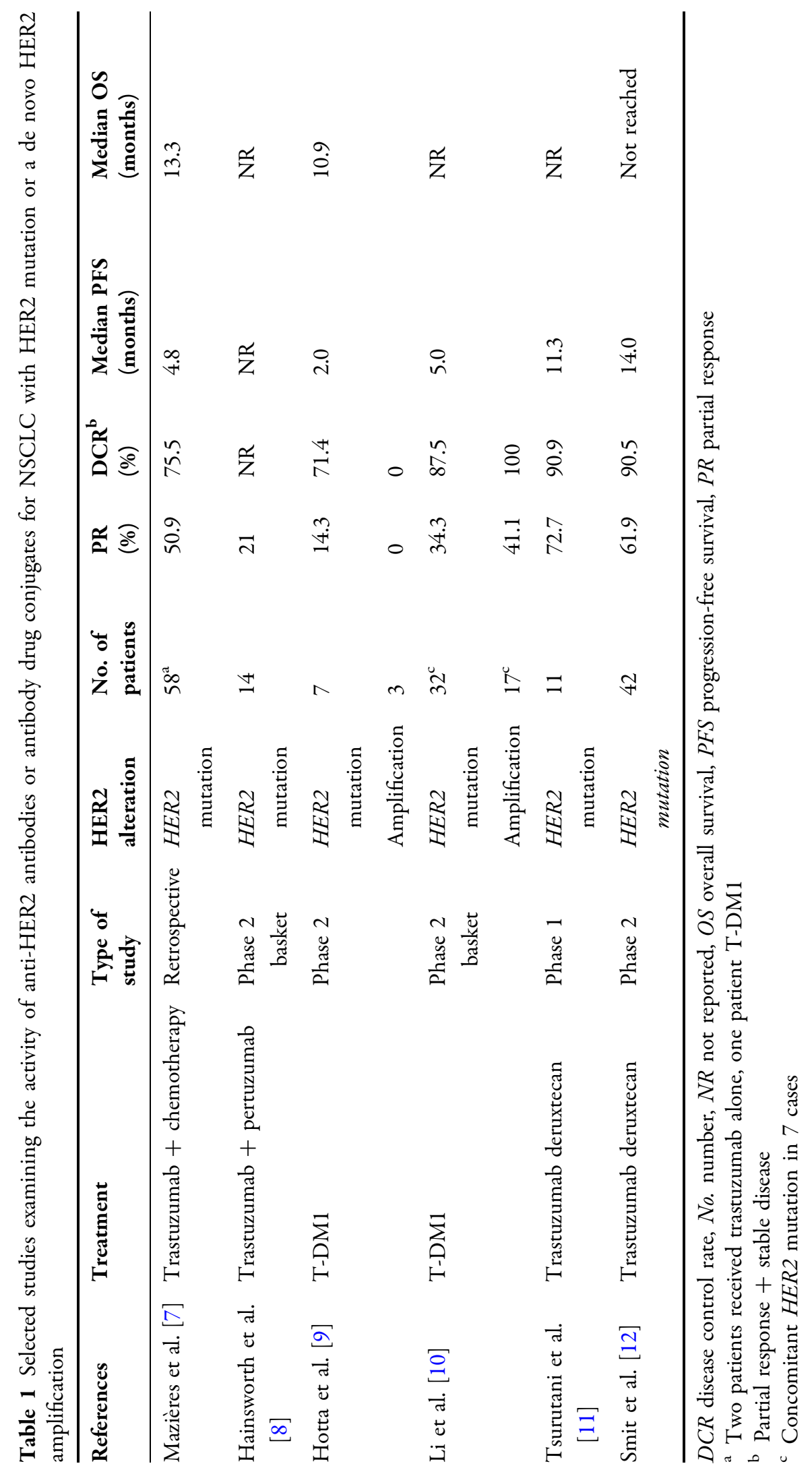


new in the management of oncogene-addicted NSCLC. As for EGFR-mutated and $A L K$-rearranged NSCLCs, targeted treatment beyond progression with an EGFR- and ALK-TKI, respectively, has been associated with some clinical benefit, especially in case of oligo-progressive disease [13, 14]. However, in EGFRmutated NSCLC, the strategy of continuing an EGFR-TKI beyond progression with the addition of cytotoxic chemotherapy has not been found superior compared with switching to chemotherapy alone [15]. On the other hand, a recent study suggested partial benefit from continuing the same ALK-TKI with the addition of chemotherapy at the time of disease progression in $A L K$-rearranged NSCLC [16]. When it comes to HER2-mutated advanced NSCLC, Li et al. observed that T-DM1 was able to induce a partial response in eight out of 18 patients, including four patients who had been pretreated with anti-HER2-targeted therapies [11]. Similarly, our case shows that continuing targeted treatment with trastuzumab beyond progression with a change in the cytotoxic companion agent at the time of progression is feasible and could be beneficial in HER2-mutated NSCLC, thus deserving consideration, especially in patients who develop acquired resistance to treatment after an initial benefit.

Unfortunately, there is a lack of studies on the type of resistance mechanisms that develop on anti-HER2 therapies in HER2-mutated NSCLC, which could be crucial in addressing whether HER2-mutated NSCLC still depends on HER2 signalling at the time of disease progression. Several barriers to the exploration of such resistance mechanisms exist. First, HER2 mutation is a relatively rare genetic alteration in NSCLC, comprising approximately $1 \%$ of all lung adenocarcinomas [1, 2]. Second, this alteration might not be universally sought, although the widespread use of next-generation sequencing (NGS), as well as the development of accurate NGS platforms for liquid biopsy, has progressively overcome this issue [17]. In fact, NGS, which allows for simultaneous testing of multiple genetic alterations, has been already implemented in several clinical settings and is much more convenient when compared with the "old" approach based on each single molecular test performed sequentially at different times. Finally, data on the efficacy of antiHER2 therapies in HER-2 mutated NSCLC are still conflicting, and these therapies may not be available in all countries, depending on local regulations governing drug access. However, we can speculate that the mechanism of resistance to anti-HER2 therapies in HER2-mutated NSCLC resemble those observed in other types of oncogene-addicted NSCLCs. For instance, resistance mechanisms in EGFR-mutated NSCLCs treated with an EGFR-TKI can be divided into on-target and off-target mechanisms, which impacts significantly the choice of the next line of therapy at the time of disease progression [18]. Similar findings have been proven to occur in case of ALK-positive disease treated with an ALK-TKI [19]. In our case, the repeat responses observed with each of the anti-HER2 therapies administered to the patient suggests the presence of on-target resistance mechanisms that might have been responsible for the benefit observed from continuous anti-HER2 blockade. However, lack of re-biopsy at the time of disease progression can only lead us to speculate on such a possibility. Certainly, clinical studies currently evaluating anti-HER2-directed therapies for HER2-mutated NSCLC need to include either tissue or liquid biopsy at the time of progression, especially in case of acquired resistance after prior response to anti-HER2 treatment, in order to increase our knowledge on the potential resistance mechanisms involved and provide useful suggestions on how to overcome them.

Interestingly, responses to anti-HER2-targeted therapies have been reported not only in the presence of HER2 mutation but also HER2 amplification [20]. De novo HER2 amplification occurs in approximately 3\% of NSCLCs and should be regarded as a different biologic event from acquired HER2 amplification in EGFRmutated patients that have been pretreated with an EGFR-TKI $[21,22]$. Table 1 lists selected studies evaluating the efficacy of anti-HER2 antibodies for HER2-amplified NSCLC. With regard to our case, we do not know whether the exceptional benefit derived from anti-HER2 therapies could be due to the simultaneous presence of HER2 amplification, as we did not 


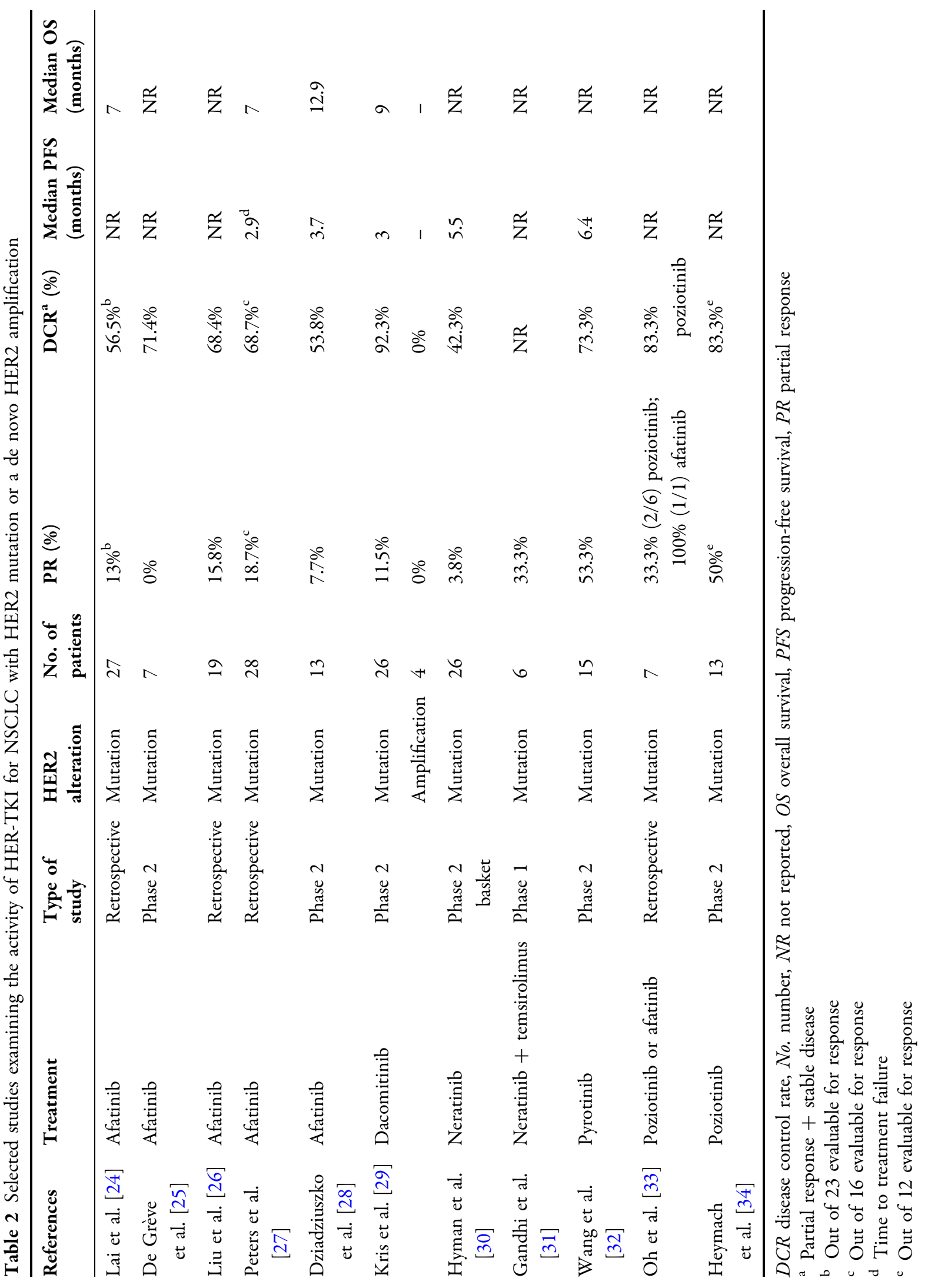


perform FISH analysis in the patient's sample collected at the time of diagnosis. However, while some overlap has been reported between HER2 overexpression and HER2 amplification, meaning that some HER2 IHC $3+$ patients are also HER2-amplified, an overlap between HER2 mutation and HER2 amplification has seldom been reported $[11,22,23]$. There have been at least two studies that have looked at the coexistence of both HER2 mutation and HER2 amplification in the same patients. On one hand, a study found a similar incidence of 3\% for each of the two molecular aberrations with none overlapping [22]. On the other, another study conducted in a large Japanese population of 1126 NSCLC patients found that HER2 amplification was found at a frequency of 5.3\% $(n=60)$, while HER2 mutation was found in $2.9 \%(n=21)$ of 724 who had an EGFR wildtype status [23]. Importantly, only one patient had a concomitant HER2 mutation and amplification, and the clinical characteristics of HER2-mutated patients (i.e. prevalence of never/light smokers and female patients) were found to be far different from those with HER2 amplification. To conclude, these data strongly suggest that, despite the fact that HER2 mutation and HER2 amplification can both result in oncogene addiction, they appear to be clearly distinct molecular features since they are unlikely to coexist in the same patient.

Importantly, a small-molecule TKI may also be beneficial in HER2-mutated NSCLC, as it was with the pan-HER-TKI poziotinib for our patient. Of note, the patient responded to poziotinib despite multiple prior lines of antiHER2 therapies. This suggests again continuous dependence on HER2 addiction of HER2-mutated NSCLC, with possible absence of cross-resistance between anti-HER2 monoclonal antibodies and pan-HER-TKI. With regard to this, other authors have reported responses to the dual EGFR/HER2-TKI afatinib in patients who have previously responded to the antiHER2 monoclonal antibodies trastuzumab and pertuzumab [24]. Table 2 lists the results with different HER-TKI administered as single agent for HER2-mutated or HER2-amplified NSCLC.

In conclusion, the present case shows that HER2 mutation is a druggable target in NSCLC, and that hitting the same target with multiple anti-HER2 therapies beyond progression could be an option in HER2-mutated NSCLC. However, ongoing clinical studies with newer antiHER2 drugs and re-biopsy at the time of disease progression are crucial in order to provide insight into the mechanisms that underlie resistance to treatment and eventually help guide the choice of the next line of therapy.

\section{ACKNOWLEDGEMENTS}

We thank the patient for giving consent to publish her case.

Funding. No funding or sponsorship was received for this study or publication of this article.

Authorship. All named authors meet the International Committee of Medical Journal Editors (ICMJE) criteria for authorship for this article and take responsibility for the integrity of the work as a whole. They have given their approval for this version to be published.

Authorship Contributions. Giulio Metro and Fausto Roila were responsible for the conception of the work, data collection and manuscript writing. Sara Baglivo, Guido Bellezza and Angelo Sidoni provided molecular data of the patient. Riccardo Moretti reviewed radiographic scans. All authors read and gave their approval for the manuscript to be published in its final version.

Disclosures. Giulio Metro, Sara Baglivo, Vienna Ludovini, Riccardo Moretti, Guido Bellezza, Angelo Sidoni, Fausto Roila have nothing to disclose.

Compliance with Ethics Guidelines. Written informed consent to publish the patient's case was provided by the patient in an anonymous form.

Data Availability. All data generated or analysed during this study are included in this published article. 
Open Access. This article is licensed under a Creative Commons Attribution-NonCommercial 4.0 International License, which permits any non-commercial use, sharing, adaptation, distribution and reproduction in any medium or format, as long as you give appropriate credit to the original author(s) and the source, provide a link to the Creative Commons licence, and indicate if changes were made. The images or other third party material in this article are included in the article's Creative Commons licence, unless indicated otherwise in a credit line to the material. If material is not included in the article's Creative Commons licence and your intended use is not permitted by statutory regulation or exceeds the permitted use, you will need to obtain permission directly from the copyright holder. To view a copy of this licence, visit http:// creativecommons.org/licenses/by-nc/4.0/.

\section{REFERENCES}

1. Jebbink M, de Langen AJ, Boelens MC, et al. The force of HER2 - a druggable target in NSCLC? Cancer Treat Rev. 2020;86:101996.

2. Jordan EJ, Kim HR, Arcila ME, et al. Prospective comprehensive molecular characterization of lung adenocarcinomas for efficient patient matching to approved and emerging therapies. Cancer Discov. 2017;7:596-609.

3. Fabi A, Metro G, Ferretti G, et al. Do HER-2 positive metastatic breast cancer patients benefit from the use of trastuzumab beyond disease progression? A mono-institutional experience and systematic review of observational studies. Breast. 2008;17: 499-505.

4. von Minckwitz G, Schwedler K, Schmidt M, et al. Trastuzumab beyond progression: overall survival analysis of the GBG 26/BIG 3-05 phase III study in HER2-positive breast cancer. Eur J Cancer. 2011;47: 2273-81.

5. Swain SM, Miles D, Kim SB, et al. Pertuzumab, trastuzumab, and docetaxel for HER2-positive metastatic breast cancer (CLEOPATRA): end-ofstudy results from a double-blind, randomised, placebo-controlled, phase 3 study. Lancet Oncol. 2020;21:519-30.
6. Davidson M, Starling N. Trastuzumab in the management of gastroesophageal cancer: patient selection and perspectives. Onco Targets Ther. 2016;9: 7235-45.

7. Mazières J, Barlesi F, Filleron $\mathrm{T}$, et al. Lung cancer patients with HER2 mutations treated with chemotherapy and HER2-targeted drugs: results from the European EUHER2 cohort. Ann Oncol. 2016;27:281-6.

8. Hainsworth JD, Meric-Bernstam F, Swanton C, et al. Targeted therapy for advanced solid tumors on the basis of molecular profiles: results from MyPathway, an open-label, phase IIa multiple basket study. J Clin Oncol. 2018;36:536-42.

9. Hotta K, Aoe K, Kozuki T, et al. A phase II study of trastuzumab emtansine in HER2-positive non-small cell lung cancer. J Thorac Oncol. 2018;13:273-9.

10. Li BT, Michelini F, Misale S, et al. HER2-mediated internalization of cytotoxic agents in ERBB2 amplified or mutant lung cancers. Cancer Discov. 2020;10:674-87.

11. Tsurutani J, Iwata H, Krop I, et al. Targeting HER2 with trastuzumab deruxtecan: a dose-expansion, phase I study in multiple advanced solid tumors. Cancer Discov. 2020;10:688-701.

12. Smit EF, Nakagawa $K$, Nagasaka $M$, et al. Trastuzumab deruxtecan (T-DXd; DS-8201) in patients with HER2-mutated metastatic non-small cell lung cancer (NSCLC): interim results of DESTINYLung01. J Clin Oncol 2020; 38(suppl; abstr 9504).

13. Park K, Yu CJ, Kim SW, et al. First-line erlotinib therapy until and beyond response evaluation criteria in solid tumors progression in Asian patients with epidermal growth factor receptor mutationpositive non-small-cell lung cancer: the ASPIRATION study. JAMA Oncol. 2016;2:305-12.

14. Ou SH, Jänne PA, Bartlett $\mathrm{CH}$, et al. Clinical benefit of continuing ALK inhibition with crizotinib beyond initial disease progression in patients with advanced ALK-positive NSCLC. Ann Oncol. 2014;25:415-22.

15. Mok TSK, Kim SW, Wu YL, et al. Gefitinib plus chemotherapy versus chemotherapy in epidermal growth factor receptor mutation-positive nonsmall-cell lung cancer resistant to first-line gefitinib (IMPRESS): overall survival and biomarker analyses. J Clin Oncol. 2017;35:4027-34.

16. Lin JJ, Schoenfeld AJ, Zhu VW, et al. Efficacy of platinum/pemetrexed combination chemotherapy in ALK-positive NSCLC refractory to second-generation ALK inhibitors. J Thorac Oncol. 2020;15: 258-65. 
17. Rolfo C, Mack PC, Scagliotti GV, et al. Liquid biopsy for advanced non-small cell lung cancer (NSCLC): a statement paper from the IASLC. J Thorac Oncol. 2018;13:1248-68.

18. O'Kane GM, Barnes TA, Leighl NB. Resistance to epidermal growth factor receptor tyrosine kinase inhibitors, T790M, and clinical trials. Curr Oncol. 2018;25(Suppl 1):S28-S37.

19. Rothenstein JM, Chooback N. ALK inhibitors, resistance development, clinical trials. Curr Oncol. 2018;25(Suppl 1):S59-S67.

20. Peters S, Stahel R, Bubendorf L, et al. Trastuzumab emtansine (T-DM1) in patients with previously treated HER2-overexpressing metastatic non-small cell lung cancer: efficacy, safety, and biomarkers. Clin Cancer Res. 2019;25:64-72.

21. Yu HA, Arcila ME, Rekhtman N, et al. Analysis of tumor specimens at the time of acquired resistance to EGFR-TKI therapy in 155 patients with EGFRmutant lung cancers. Clin Cancer Res. 2013;19: 2240-7.

22. Li BT, Ross DS, Aisner DL, et al. HER2 amplification and HER2 mutation are distinct molecular targets in lung cancers. J Thorac Oncol. 2016;11:414-9.

23. Ninomiya $\mathrm{K}$, Hata $\mathrm{T}$, Yoshioka $\mathrm{H}$, et al. A prospective cohort study to define the clinical features and outcome of lung cancers harboring HER2 aberration in Japan (HER2-CS STUDY). Chest. 2019;156: 357-66.

24. Lai WV, Lebas L, Barnes TA, et al. Afatinib in patients with metastatic or recurrent HER2-mutant lung cancers: a retrospective international multicentre study. Eur J Cancer. 2019;109:28-35.

25. De Grève J, Moran T, Graas MP, et al. Phase II study of afatinib, an irreversible ErbB family blocker, in demographically and genotypically defined lung adenocarcinoma. Lung Cancer. 2015;88:63-9.

26. Liu Z, Wu L, Cao J, et al. Clinical characterization of ERBB2 exon 20 insertions and heterogeneity of outcomes responding to afatinib in Chinese lung cancer patients. Onco Targets Ther. 2018;11: 7323-31.

27. Peters S, Curioni-Fontecedro A, Nechushtan $H$, et al. Activity of afatinib in heavily pretreated patients with ERBB2 mutation-positive advanced NSCLC: findings from a global named patient use program. J Thorac Oncol. 2018;13:1897-905.

28. Dziadziuszko R, Smit EF, Dafni U, et al. Afatinib in NSCLC with HER2 mutations: results of the prospective, open-label phase II NICHE trial of European Thoracic Oncology Platform (ETOP). J Thorac Oncol. 2019;14:1086-94.

29. Kris MG, Camidge DR, Giaccone G, et al. Targeting HER2 aberrations as actionable drivers in lung cancers: phase II trial of the pan-HER tyrosine kinase inhibitor dacomitinib in patients with HER2mutant or amplified tumors. Ann Oncol. 2015;26: 1421-7.

30. Hyman DM, Piha-Paul SA, Won H, et al. HER kinase inhibition in patients with HER2- and HER3-mutant cancers. Nature. 2018;554:189-94.

31. Gandhi L, Bahleda R, Tolaney SM, et al. Phase I study of neratinib in combination with temsirolimus in patients with human epidermal growth factor receptor 2-dependent and other solid tumors. J Clin Oncol. 2014;32:68-75.

32. Wang Y, Jiang T, Qin Z, et al. HER2 exon 20 insertions in non-small-cell lung cancer are sensitive to the irreversible pan-HER receptor tyrosine kinase inhibitor pyrotinib. Ann Oncol. 2019;30:447-55.

33. Oh IJ, Hur JY, Park CK, et al. Clinical activity of pan-HER inhibitors against HER2-mutant lung adenocarcinoma. Clin Lung Cancer. 2018;19: e775-e78181.

34. Heymach J, Negrao R, et al. A phase II trial of poziotinib in EGFR and HER2 exon 20 mutant nonsmall cell lung cancer (NSCLC). J Thorac Oncol. 2018;13:S323-S324324 (abstract). 